\title{
Integrated Framework for Selection of Additive and Nonadditive Genetic Markers for Genomic Selection
}

\author{
SAYANTI GUHA MAJUMDAR, ${ }^{*}$ ANIL RAI, ${ }^{*}$ and DWIJESH C. MISHRA
}

\begin{abstract}
Genomic selection is a modified form of marker-assisted selection in which the markers from the whole genome are used to estimate the genomic-estimated breeding value (GEBV). Several estimators are available to estimate GEBV. These estimators are able to capture either additive genetic effects or nonadditive genetic effects. However, there is hardly any procedure available that could capture both the effects simultaneously. Therefore, this study has been conducted to develop an integrated framework that is able to capture both additive and nonadditive effects efficiently. This integrated framework has been developed after evaluating existing additive and nonadditive models for marker selection. Furthermore, two efficient additive and nonadditive methods, that is, sparse additive models (SpAM) and Hilbert-Schmidt independence criterion least absolute shrinkage and selection operator (HSIC LASSO), have been combined to select both additive and nonadditive genetic markers for estimation of GEBV. The performance of the proposed framework has been evaluated on the basis of prediction accuracy, fraction of correctly selected features, and redundancy rate, along with standard error of mean for estimation of GEBV, compared with the individual performances of SpAM and HSIC LASSO separately. The newly developed framework is found to be satisfactory in terms of its performance and found to be robust for estimation of GEBV.
\end{abstract}

Keywords: additive and nonadditive models, genetic markers, GEBV, genomic selection, prediction accuracy, redundancy rate.

\section{INTRODUCTION}

G ENOMIC SELECTION (GS) was proposed by Meuwissen et al. (2001) as an improved variant of markerassisted selection for efficient breeding of animals and plants. Marker information covering whole genome is used to estimate the breeding value in GS, even without information of exact gene location. With the advancement of next-generation sequencing technology, it is now easier to genotype single-nucleotide polymorphisms (SNPs) within a short period of time, which is being used for GS of superior parents based on genomic-estimated breeding value (GEBV). To estimate GEBV, phenotypic and genotypic information of

Centre for Agricultural Bioinformatics, ICAR-Indian Agricultural Statistics Research Institute, Pusa, New Delhi, India.

*These authors contributed equally to this work. 
the reference population is collected to build a prediction model. Initially, all the individuals in the reference population are genotyped to get SNPs from the entire genome. The precision of prediction model increases by increasing the sample size of reference population. The collected information on genotype and phenotype from reference population is used to train a predictive model for estimation of GEBV. This developed model is being used to obtain GEBV of an individual from the breeding population based on marker information.

Many models are available in literature for selection of genetic markers. Most of them are developed to capture additive genetic effects. However, some models are also available, which can be used for modeling nonadditive genetic effects, that is, epistasis. In general, it has been observed that these models are able to efficiently capture either the additive or nonadditive genetic effects. Selection of appropriate genetic markers is an important step in GS for estimation of GEBV. The selection of most relevant markers depends on adopting the most efficient feature selection method and the estimation of GEBV for trait of interest. There is hardly any model in literature that is capable to capture both additive and nonadditive genetic effects efficiently. Therefore, there is a strong need to appropriately develop an integrated framework for selection of the most appropriate additive and nonadditive genetic markers for estimation of GEBV. Therefore, first, we have evaluated statistical performance of important models for selection of additive effect markers, namely, linear least-squared regression (Meuwissen et al., 2001), least absolute shrinkage and selection operator (LASSO; Tibshirani, 1996), ridge regression (Hoerl and Kennard, 1970a,b), BLUP (best linear unbiased prediction) (Henderson, 1975), sparse additive models (SpAM; Ravikumar et al., 2009) along with two important procedures for selection of nonadditive genetic markers, namely, mRMR (minimum redundancy maximum relevance) (Peng et al., 2005) and Hilbert-Schmidt Independence Criterion (HSIC) LASSO (Yamada et al., 2014) through a simulation study.

In this empirical study, it was observed that SpAM performs very well for additive genetic architecture and HSIC LASSO is comparatively efficient in case of an epistatic effect in data for selection of genetic markers. It may be noted that, in the presence of an epistatic effect, the performance of SpAM is very poor, whereas for additive genetic effect, the performance of HSIC LASSO is encouraging. Keeping in view these facts, it is pertinent to develop a robust framework for selection of genetic markers for estimation of GEBV for GS, which can account for both additive and epistatic genetic effects in selection of parents from the breeding population. In this article, an attempt has been made to propose an integrated framework for selection of genetic markers to estimate GEBV. The comparative performance of the proposed integrated framework has been examined through a simulation study.

\section{METHODS}

An integrated framework for selection of genetic markers to estimate GEBV has been developed for GS by combining selected genetic markers from an additive model, that is, SpAM and a nonadditive model, that is, HSIC LASSO as these models are found to be superior for selection of additive and nonadditive genetic markers, respectively. In the first instance, a brief description of SpAM and HSIC LASSO models is given below.

\subsection{Sparse additive models}

The SpAM is useful for high-dimensional feature selection (Liu et al., 2009; Ravikumar et al., 2009; Raskutti et al., 2012; Suzuki and Sugiyama, 2013). The SpAM optimization problem can be expressed as follows:

$$
\min _{\beta_{1}, \ldots, \beta_{p} \in \mathbb{R}^{n}}\left\|y-\sum_{k=1}^{p} K^{(k)} \beta_{k}\right\|_{2}^{2}+\lambda \sum_{k=1}^{p} \sqrt{\frac{1}{n}\left\|K^{(k)} \beta_{k}\right\|_{2}^{2}}
$$

where $n$ is the total number of individuals, $p$ is the total number of variables (i.e., markers), $y$ is the vector of phenotypic value for all the individuals, $\beta_{k}=\left[\beta_{k, 1}, \ldots, \beta_{k, n}\right]^{T}, k=1, \ldots, p$ are regression coefficient vectors, $K_{i, j}^{(k)}=K\left(x_{k, i}, x_{k, j}\right)$ is gram matrix, and $i, j=1, \ldots, n, x$ are the elements of the incidence matrix, $K\left(x, x^{\prime}\right)$ is kernel function, $\beta_{k, j}$ is a coefficient for $\left[K\left(x_{k, 1}, x_{k, j}\right), \ldots, K\left(x_{k, n}, x_{k, j}\right)\right]^{T}$, and $\lambda>0$ is a regularization parameter. SpAM is similar to the hierarchical multiple kernel learning (Bach, 2009), which implements an SpAM with an alternative sparsity-inducing regularization. SpAM is a convex method and can be efficiently optimized by the back-fitting algorithm. 
A disadvantage of SpAM is that it can only deal with additive effects. In case of epistatic effects in the data, SpAM may fail to select significant markers. Another disadvantage is that the output y should be a real number in SpAM, which indicates that SpAM cannot deal with structured outputs such as multilabel and graph data. Moreover, SpAM is a computationally expensive procedure.

\subsection{HSIC least absolute shrinkage and selection operator}

A feature-wise nonlinear LASSO of the following form was proposed by Yamada et al. (2013), which is called as HSIC (Gretton et al., 2005) LASSO.

$$
\begin{gathered}
\min _{\boldsymbol{\beta} \in \mathbb{R}^{n}} \quad \frac{1}{2}\left\|\overline{\boldsymbol{L}}-\sum_{k=1}^{p} \beta_{k} \overline{\boldsymbol{K}}^{(k)}\right\|_{\text {Frob }}^{2}+\lambda\|\boldsymbol{\beta}\|_{1} \\
\text { s.t. } \beta_{1}, \ldots, \beta_{p} \geq 0,
\end{gathered}
$$

where $n$ is the total number of individuals, $p$ is the total number of variables (markers), $\|\cdot\|_{\text {Frob }}$ is the Frobenius norm, $\overline{\boldsymbol{K}}^{(k)}=\Gamma \boldsymbol{K}^{(k)} \Gamma$ and $\overline{\boldsymbol{L}}=\Gamma \boldsymbol{L} \Gamma$ are centered gram matrices, $\boldsymbol{K}_{i, j}^{(k)}=K\left(x_{k, i}, x_{k, j}\right)$ and $L_{i, j}=L\left(y_{i}, y_{j}\right)$ are gram matrices, $x_{k, i}$ and $x_{k, j}$ are the elements of the incidence matrix, $y_{i}$ and $y_{j}$ are the elements of the vector of phenotypic value for all the individuals, $K\left(x, x^{\prime}\right)$ and $L\left(y, y^{\prime}\right)$ are kernel functions, $\Gamma=\mathbf{I}_{n}-\frac{1}{2} 1_{n} 1_{n}^{\mathrm{T}}$ is the centering matrix, $\mathbf{I}_{n}$ is the $n$-dimensional identity matrix, and $1_{n}$ is the $n$-dimensional vector with all ones. Here, a non-negativity constraint is used so that meaningful markers are selected. As output, gram matrix $\boldsymbol{L}$ is used to select markers in HSIC LASSO, and it is possible to naturally incorporate structured outputs via kernels. Moreover, we can perform feature selection even if the training data set consists of input $x$ and its affinity information $\boldsymbol{L}$ link structures between inputs. Differences from the original formulation of LASSO are that, in this case, kernel functions $K$ and $L$ are different and nonnegativity constraint is imposed. The first term in this equation means that we are regressing the output kernel matrix $\overline{\boldsymbol{L}}$ by a linear combination of feature-wise input kernel matrices $\left\{\overline{\boldsymbol{K}}^{(k)}\right\}_{k=1}^{p}$.

\subsection{Proposed integrated framework for marker selection}

In this study, Linear least-squared regression (Meuwissen et al., 2001), LASSO (Tibshirani, 1996), ridge regression (Hoerl and Kennard, 1970), BLUP (Henderson, 1975), SpAM (Ravikumar et al., 2009), mRMR (Peng et al., 2005), and HSIC LASSO (Yamada et al., 2013) have been evaluated through a simulation study for identification of additive and nonadditive genetic markers based on prediction accuracy (PA), fraction of correctly selected features, and redundancy rate (RED). It was found that SpAM and HSIC LASSO are reasonably superior for identification of additive and nonadditive genetic markers (Guha Majumdar et al., in press). Therefore, it is proposed to estimate GEBV for GS based on additive and nonadditive genetic markers identified through these procedures. To estimate GEBV following an integrated model is proposed:

$$
y_{\text {Int }}=w y_{S p}+(1-w) y_{H L}
$$

where $y_{i n t}$ is the predicted phenotype (GEBV) of the integrated model, $w$ is $\frac{\sigma_{H L}^{2}}{\sigma_{S L}^{2} \sigma_{H L}^{2}}$, where $\sigma_{H L}^{2}$ and $\sigma_{S p}^{2}$ are the error variances of models HSIC LASSO and SpAM, respectively, $y_{S p}$ is the predicted GEBV from SpAM and $y_{H L}$ is the GEBV from HSIC LASSO. Let the error variance of $y_{I n t}$ be denoted by $\sigma_{I n t}^{2}$. The $\sigma_{I n t}^{2}$ can be written after optimizing $w$, as follows:

$$
\begin{gathered}
\sigma_{\text {Int }}^{2}=\left(\frac{\sigma_{H L}^{2}}{\sigma_{S p}^{2}+\sigma_{H L}^{2}}\right)^{2} \sigma_{S p}^{2}+\left(\frac{\sigma_{S p}^{2}}{\sigma_{S p}^{2}+\sigma_{H L}^{2}}\right)^{2} \sigma_{H L}^{2} \\
=\frac{\sigma_{S p}^{2} \sigma_{H L}^{2}}{\sigma_{S p}^{2}+\sigma_{H L}^{2}}
\end{gathered}
$$

The estimation of the above error variance is highly complex in nature. Therefore, it is proposed to estimate this by following the approach of Fan et al. (2012). The detail of the same is described in the following section. 


\subsection{Estimation of error variance of proposed integrated framework}

There are several methods to estimate error variances $\sigma_{S p}^{2}$ and $\sigma_{H L}^{2}$. In this study, we have considered the refitted cross-validation (RCV) method of error variance estimation (Fan et al., 2012). The algorithm of the $\mathrm{RCV}$ method is described below:

i. Consider a data set $\left(\mathbf{X}_{n \times p}, \mathbf{Y}_{n \times 1}\right)$. We denote it as $\mathbf{S}$.

ii. $\mathbf{S}$ is divided into two groups randomly, each subsample having size of $n / 2$.

$$
\mathbf{S}=\left\{\mathbf{S}^{(1)}, \mathbf{S}^{(2)}\right\}
$$

where $\mathbf{S}^{(1)}=\left(\mathbf{X}_{(n / 2) \times p}^{(1)}, \mathbf{Y}_{(n / 2) \times 1}^{(1)}\right)$ and $\mathbf{S}^{(2)}=\left(\mathbf{X}_{(n / 2) \times p}^{(2)}, \mathbf{Y}_{(n / 2) \times 1}^{(2)}\right)$

iii. Variable selection has been performed on $\mathbf{S}^{(1)}$ using SpAM or HSIC LASSO and let $\mathbf{M}^{(1)}$ be a set of selected variables from subsample $\mathbf{S}^{(1)}$.

iv. Using only the selected variables, error variance is estimated through $\mathbf{S}^{(2)}$ with ordinary least-squares estimation.

$$
\hat{\sigma}^{(2)^{2}}=\frac{\mathbf{Y}^{(2)^{T}}\left(\mathbf{I}_{\frac{n}{2}}-\mathbf{P}_{\mathbf{M}^{(1)}}^{(2)}\right) \mathbf{Y}^{(2)}}{\frac{n}{2}-\left|\mathbf{M}^{(1)}\right|}
$$

where $\mathbf{P}_{\mathbf{M}^{(1)}}^{(2)}=\mathbf{X}_{\mathbf{M}^{(1)}}^{(2)}\left(\mathbf{X}_{\mathbf{M}^{(1)}}^{(2)}{ }^{T} \mathbf{X}_{\mathbf{M}^{(1)}}^{(2)}\right)^{-1} \mathbf{X}_{\mathbf{M}^{(1)}}^{(2)} T$

v. In the next stage, variable selection has been performed on $\mathbf{S}^{(2)}$ using SpAM or HSIC LASSO and $\mathbf{M}^{(2)}$ is a set of selected variables from subsample $\mathbf{S}^{(2)}$.

vi. Using only the selected variables, variance is estimated in $\mathbf{S}^{(1)}$ with ordinary least-squares estimation.

$$
\hat{\sigma}^{(1)^{2}}=\frac{\mathbf{Y}^{(1)^{T}}\left(\mathbf{I}_{\frac{n}{2}}-\mathbf{P}_{\mathbf{M}^{(2)}}^{(1)}\right) \mathbf{Y}^{(1)}}{\frac{n}{2}-\left|\mathbf{M}^{(2)}\right|}
$$

where $\mathbf{P}_{\mathbf{M}^{(2)}}^{(1)}=\mathbf{X}_{\mathbf{M}^{(2)}}^{(1)}\left(\mathbf{X}_{\mathbf{M}^{(2)}}^{(1)} \mathbf{X}_{\mathbf{M}^{(2)}}^{(1)}\right)^{-1} \mathbf{X}_{\mathbf{M}^{(2)}}^{(1)} T$

vii. The final estimator of variance is

$$
\hat{\sigma}_{R C V}^{2}=\frac{\hat{\sigma}^{(1)^{2}}+\hat{\sigma}^{(2)^{2}}}{2}
$$

Here, $\hat{\sigma}_{R C V}^{2}$ represents both $\sigma_{S p}^{2}$ and $\sigma_{H L}^{2}$. This means both $\sigma_{S p}^{2}$ and $\sigma_{H L}^{2}$ will be obtained by following the above algorithm of RCV. Let error variance estimator of SpAM or HSIC LASSO be represented by $\hat{\sigma}_{S p}^{2}$ and $\hat{\sigma}_{H L}^{2}$, respectively.

Then, estimator of error variance of integrated model, that is, $\hat{\sigma}_{I n t}^{2}$, is given by substituting $\sigma_{S p}^{2}$ and $\sigma_{H L}^{2}$ with estimated values of $\sigma_{S p}^{2}$ and $\sigma_{H L}^{2}$, that is, $\hat{\sigma}_{S p}^{2}$ and $\hat{\sigma}_{H L}^{2}$ in expression (iv) of $\sigma_{I n t}^{2}$.

\subsection{Simulation study}

A genome with 10 chromosomes with specified length was simulated using R package QTL (quantitative trait locus) Bayesian interval mapping ("qtlbim"; Yandell et al., 2007; Yandell et al., 2012). The package qtlbim follows Cockerham's model for simulating quantitative trait loci with epistasis (Kao and Zeng, 2002). The Cockerham's model for quantitative trait was controlled by two epistatic genes A and $\mathrm{B}$, from a sample of size $n$ of an F2 population, and the trait value of the $k^{\text {th }}$ individual with genotype $i j$ can be described as follows:

$$
y_{i j k}=G_{i j}+\varepsilon_{i j k}=\mu+a_{1} x_{1}+d_{1} z_{1}+a_{2} x_{2}+d_{2} z_{2}+i_{a a} w_{a a}+i_{a d} w_{a d}+i_{d a} w_{d a}+i_{d d} w_{d d}+\varepsilon_{i j k}
$$

where $G_{i j}$ denotes the genotypic value of the genotype $i j, \mu$ is the mean, $a_{1}$ is the additive effect of locus A, $d_{1}$ is the dominance effect of locus A, $a_{2}$ is the additive effect of locus $\mathrm{B}, d_{2}$ is the dominance effect of locus 
$\mathrm{B}, i_{a a}$ is additive $\times$ additive effect of loci $\mathrm{A}$ and $\mathrm{B}, i_{a d}$ is additive $\times$ dominance effect of loci $\mathrm{A}$ and $\mathrm{B}, i_{d a}$ is dominance $\times$ additive effect of loci $\mathrm{A}$ and $\mathrm{B}$, and $i_{d d}$ is dominance $\times$ dominance effect of loci $\mathrm{A}$ and $\mathrm{B}$. The coded variables are defined as follows:

$$
\begin{aligned}
& x_{1}=\left\{\begin{array}{rl}
1 & \text { if } A \text { is } A A \\
0 & \text { if } A \text { is } A a \\
-1 & \text { if } A \text { is } a a
\end{array} \quad x_{2}=\left\{\begin{aligned}
1 & \text { if } B \text { is } B B \\
0 & \text { if } B \text { is } B b \\
-1 & \text { if } B \text { is } b b
\end{aligned}\right.\right. \\
& z_{1}=\left\{\begin{array}{rl}
1 / 2 & \text { if } A \text { is } A a \\
-1 / 2 & \text { otherwise }
\end{array} \quad z_{2}=\left\{\begin{array}{cc}
1 / 2 & \text { if } B \text { is } B b \\
-1 / 2 & \text { otherwise }
\end{array}\right.\right. \\
& w_{a a}=x_{1} \times x_{2} \quad w_{a d}=x_{1} \times z_{2} \quad w_{d a}=z_{1} \times x_{2} \quad w_{d d}=z_{1} \times z_{2}
\end{aligned}
$$

and $\varepsilon_{i j k}$ is residual of the $k^{\text {th }}$ individual with genotype $i j$.

The genome contains 1000 markers, which were equally spaced over the chromosomes and each chromosome has 100 markers. The phenotypic values are normally distributed. For the additive model, there is one QTL in each chromosome with either a positive or negative additive effect and no epistatic interaction. For the epistatic model, we assumed two QTLs on each of the five chromosomes and the remaining five chromosomes have no QTL resulting in 5 two-way epistatic interactions. In this way, 24 different data sets are generated with genotypic and phenotypic information for the F2 population. Each data set contains 200 individuals and 1000 biallelic markers. Each data set has a different combination of heritability (narrow sense) and epistatic effect. Details of simulated data sets are given in Table 1. For each data set with a specific genetic architecture, 50 replicates were generated and from each replicate, $80 \%$ individuals were selected randomly for training and the remaining $20 \%$ individuals are kept for testing, that is, to predict phenotype (GEBV). Again for each of these 50 replicates, this random selection of training/testing data sets was repeated 50 times; this results in 2500 training/testing data sets in total for a specific genetic architecture.

From these simulated data sets, GEBV was estimated using above models, that is, SpAM, HSIC LASSO, and proposed integrated model framework using R (R Core Team, 2017). For SpAM, we have used SAM package (Zhao et al., 2014) and applied samQL function with default parameter values. Ten highly significant markers have been selected and used for the prediction of GEBV of the testing data set with the help of predict function using SpAM fitted model on the training data set. For HSIC LASSO, a function in $\mathrm{R}$ has been developed to kernelize the input variable matrix and output vector. Then, the penalized function of penalized package (Goeman et al., 2010) has been used to fit this kernelized LASSO model and 10 highly significant markers were selected to predict phenotype (GEBV) from testing data set using predict function. Furthermore, to predict the GEBV from the proposed integrated model framework, R code has been written to combine the selected markers from these two models.

Then the performance of SpAM, HSIC LASSO, and the proposed integrated model framework has been evaluated on the basis of PA, fraction of correctly selected features (F), and the RED. PA can be described

Table 1. Description of Genetic Architecture of Simulated Data Sets

\begin{tabular}{lccccc}
\hline $\begin{array}{l}\text { S. no. of } \\
\text { data set }\end{array}$ & $\begin{array}{c}\text { Heritability } \\
\left(\mathrm{h}^{2}\right)\end{array}$ & $\begin{array}{c}\text { Epistatic } \\
\text { effect }(\mathrm{E})\end{array}$ & $\begin{array}{c}\text { S. no. of } \\
\text { data set }\end{array}$ & $\begin{array}{c}\text { Heritability } \\
\left(\mathrm{h}^{2}\right)\end{array}$ & $\begin{array}{c}\text { Epistatic } \\
\text { effect }(\mathrm{E})\end{array}$ \\
\hline 1 & 0.1 & 0 & 13 & 0.5 & 0 \\
2 & 0.1 & 5 & 14 & 0.5 & 5 \\
3 & 0.1 & 10 & 15 & 0.5 & 10 \\
4 & 0.1 & 15 & 16 & 0.5 & 15 \\
5 & 0.2 & 0 & 17 & 0.7 & 0 \\
6 & 0.2 & 5 & 18 & 0.7 & 5 \\
7 & 0.2 & 10 & 19 & 0.7 & 10 \\
8 & 0.2 & 15 & 20 & 0.7 & 15 \\
9 & 0.3 & 0 & 21 & 0.9 & 0 \\
10 & 0.3 & 5 & 22 & 0.9 & 5 \\
11 & 0.3 & 10 & 23 & 0.9 & 10 \\
12 & 0.3 & 15 & 24 & 0.9 & 15 \\
\hline
\end{tabular}


as the correlation between the actual phenotypic $\left(y_{\text {actual }}\right)$ values and the predicted phenotypic $\left(y_{\text {pred }}\right)$ values (Howard et al., 2014).

$$
\text { Prediction accuracy }(P A)=\operatorname{correlation}\left(y_{\text {actual }}, y_{\text {pred }}\right)
$$

The RED score (Zhao et al., 2010) is obtained by the following:

$$
R E D=\frac{1}{m(m-1)} \sum_{u_{k}, u_{j}, k>l}\left|\rho_{k, l}\right|
$$

where $\rho_{k, l}$ is the correlation coefficient between the $k$-th and $l$-th markers. A high RED score implies that selected markers are strongly correlated to each other, which means many redundant markers are selected. So, lower RED is desirable for feature selection.

Fraction of correctly selected features (Yamada et al., 2013) is expressed by the following:

$$
F=\frac{N_{c}}{N_{t}}
$$

where $F$ is fraction of correctly selected features, $N_{c}$ is the number of correctly selected features, and $N_{t}$ is the number of total selected features.

\section{RESULTS}

The error variance estimation method RCV has been applied to the simulated data sets using R platform. The values of the error variances of SpAM, HSIC LASSO, and integrated model framework are given in Table 2.

Table 2. Estimated Error Variances of Genomic Estimated Breeding VAlue From Different Models

\begin{tabular}{lrrrc}
\hline & & & & \\
$\mathrm{h}^{2}$ & $\mathrm{E}$ & $\begin{array}{c}\text { Variance } \\
(\text { SpAM })\end{array}$ & $\begin{array}{c}\text { Variance } \\
(\text { HSIC })\end{array}$ & $\begin{array}{c}\text { Variance } \\
\text { (integrated model } \\
\text { framework) }\end{array}$ \\
\hline 0.1 & 0 & 2.2066 & 10.9564 & 1.8296 \\
& 5 & 39.8688 & 7.8753 & 6.5603 \\
& 10 & 135.5452 & 6.7217 & 6.3996 \\
& 15 & 308.8736 & 6.5920 & 6.4514 \\
0.2 & 0 & 2.1280 & 11.7579 & 1.7888 \\
& 5 & 36.5749 & 7.8641 & 6.4561 \\
& 10 & 130.5592 & 6.4878 & 6.1760 \\
& 15 & 289.8784 & 6.5585 & 6.4107 \\
0.3 & 0 & 2.2372 & 11.7919 & 1.8755 \\
& 5 & 37.2135 & 7.7047 & 6.3677 \\
& 10 & 131.3778 & 5.7770 & 5.5288 \\
& 15 & 291.9679 & 5.7645 & 5.6502 \\
0.5 & 0 & 5.1772 & 10.6224 & 3.4664 \\
& 5 & 40.2630 & 7.0549 & 5.9847 \\
& 10 & 147.9817 & 5.1230 & 4.9488 \\
& 15 & 314.9257 & 4.1673 & 4.112 \\
0.7 & 0 & 14.6155 & 8.7070 & 5.4266 \\
& 5 & 43.2414 & 6.9314 & 5.9625 \\
& 10 & 164.7886 & 4.4756 & 4.3567 \\
& 15 & 336.0471 & 4.0835 & 4.0336 \\
0.9 & 0 & 71.0053 & 6.2260 & 5.7151 \\
& 5 & 98.9779 & 4.5325 & 4.3300 \\
& 10 & 173.3728 & 4.5618 & 4.4424 \\
& 15 & 358.5543 & 3.4429 & 3.4100 \\
\hline
\end{tabular}

HSIC, Hilbert-Schmidt independence criterion; SpAM, sparse additive models. 
Table 2 shows the estimated error variances of GEBV estimates obtained from different models. The following points may be noted from the results of Table 2 :

i. The performance of SpAM for selecting genetic markers and estimation of GEBV, with respect to variance, is better than HSIC LASSO for low and medium heritability level, that is, $h^{2}$ ranging from 0.1 to 0.5 with no epistatic effect. However, in case of higher heritability level, that is, above 0.6 , the performance of HSIC LASSO is far superior than SpAM when there is no epistatic effect. Furthermore, the variance of SpAM increases by increasing the level of epistatic effect, whereas the variance of HSIC LASSO generally decreases when epistatic effect increases.

ii. The variance of HSIC LASSO is much lower than the variance of SpAM in the presence of epistatic effect (ranging from 5 to 15) irrespective of heritability levels. This shows that the performance of HSIC LASSO is far superior to SpAM for estimation of GEBV when epistatic effect is present in a genetic architecture.

iii. The variance of proposed integrated model for estimation of GEBV is always less than the variance of HSIC LASSO and SpAM irrespective of heritability level and level of epistatic effects. This clearly indicates that the proposed integrated model performs very well in all genetic architectures.

Also, the performances of each model for selection of genetic markers and estimation of GEBV on the basis of PA, fraction of correctly selected features (F), and RED, along with their standard error (SE) of mean, are given in Supplementary Table S1.

The relationship among PA, F, and RED is presented in Figures 1 to 3 for different levels of heritability and epistatic effects. The following points may be noted from these figures.
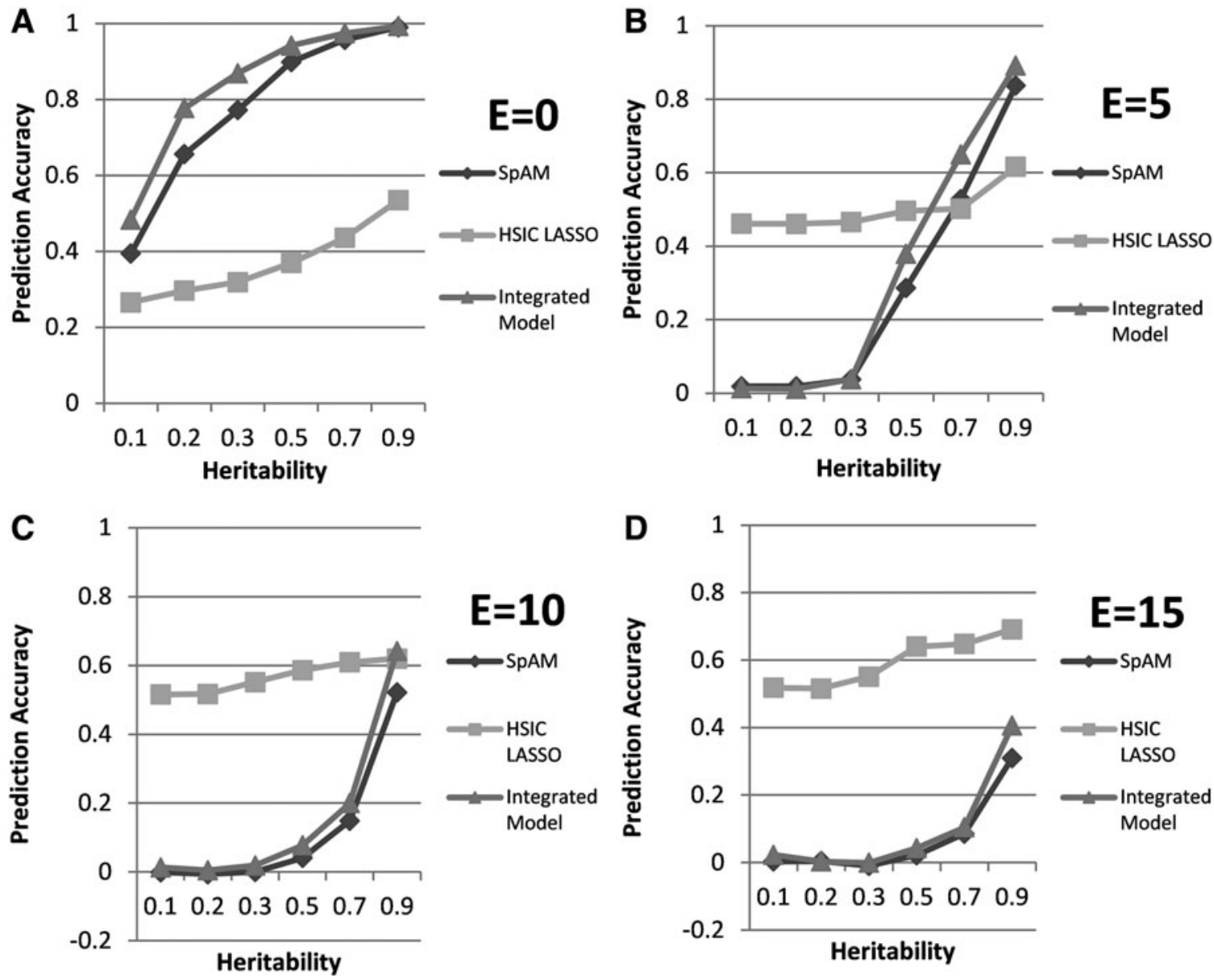

FIG. 1. Comparison of prediction accuracy with different levels of heritability $\left(h^{2}\right)$ and epistatic effect $(E)$. HSIC, Hilbert-Schmidt independence criterion; LASSO, least absolute shrinkage and selection operator; SpAM, sparse additive models. $\mathbf{A}, \mathrm{E}=0 ; \mathbf{B}, \mathrm{E}=5 ; \mathbf{C}, \mathrm{E}=10 ; \mathbf{D}, \mathrm{E}=15$. 
i. Figure 1 shows the relationship between heritability and PA for different levels of epistatic effects. From this figure, it can be observed that the developed integrated model outperforms in case of PA when the epistatic effect is 0 , that is, when the genetic effect is additive in nature (Fig. 1a). When the epistatic effect is 5, the PA of integrated model based on selected additive and nonadditive genetic markers for low heritability is poor, but for a higher level of heritability such as 0.7 and 0.9 , the performance of the proposed integrated model is superior to SpAM and HSIC LASSO (Fig. 1b). However, in case of higher epistatic effects such as 10 and 15, the performance of the proposed integrated model is better than SpAM but inferior than HSIC LASSO (Fig. 1c, d).

ii. The relationship between different levels of heritability and fraction of correctly selected features is depicted in Figure 2. It can be observed from this figure that SpAM is able to select correctly the maximum number of markers even at low levels of heritability when the epistatic effect is 0 and able to select all features correctly when the heritability level is above 0.3 in the absence of epistatic effect (Fig. 2a). In case of low epistatic effect, that is, $E=5$, the performance of all three models considering this study with respect to $\mathrm{F}$ is poor for low heritability, that is, $h^{2}$ ranging from 0 to 0.3 , however, for higher heritability the performance of SpAM is best, which is closely followed by a proposed model (Fig. 2b). However, in case of high epistatic effects, the performance of the proposed model was found to be better than SpAM and HSIC LASSO (Fig. 2c, d).

iii. Figure 3 presents the relationship between heritability and RED for different levels of epistatic effect. It can be seen from this figure that RED score for all three methods, that is, SpAM, HSIC LASSO, and proposed model is very low, that is, $<0.06$, irrespective of level of heritability and epistatic effect. This clearly indicates that all three methods considered in this study are able to remove
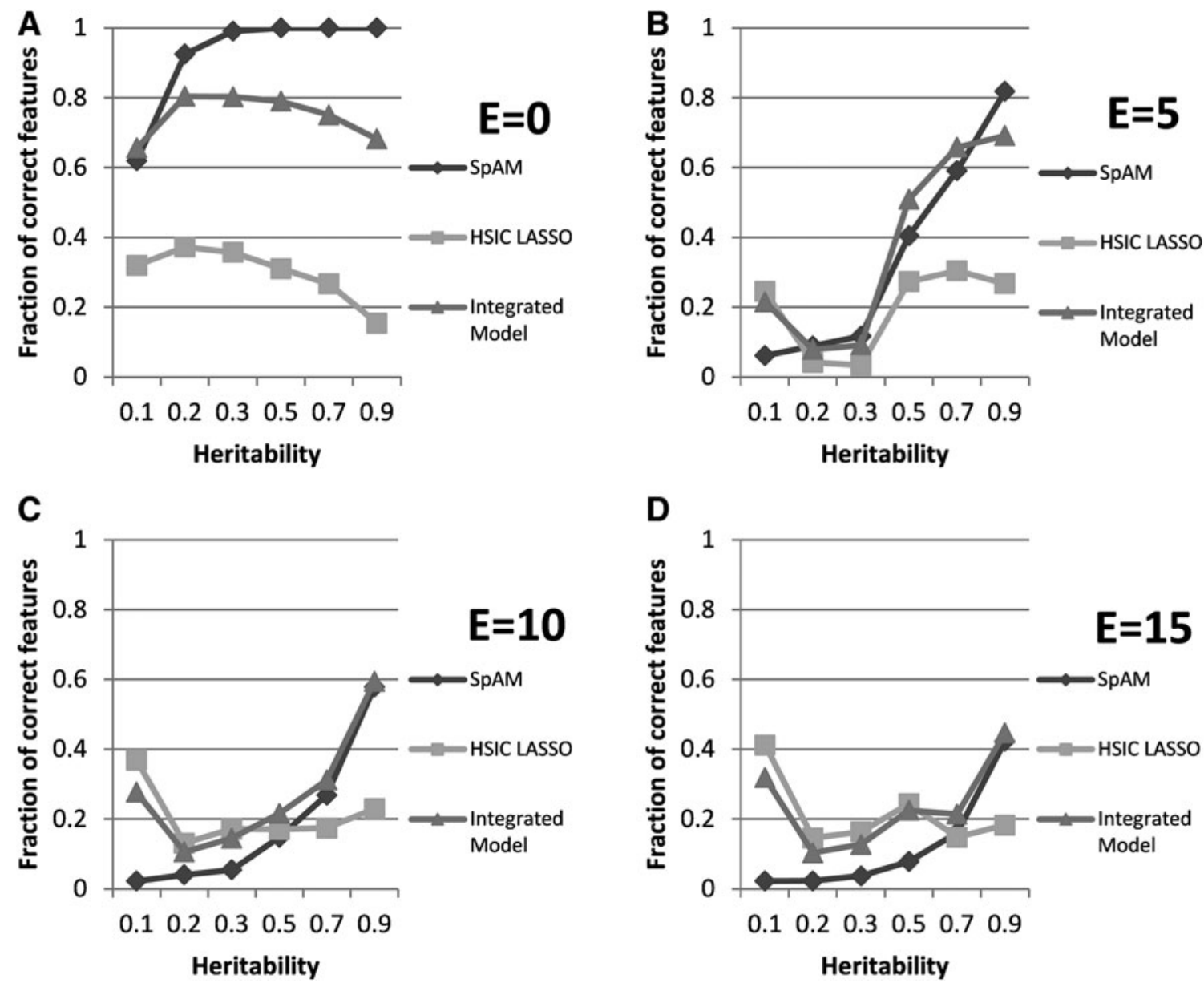

FIG. 2. Comparison of fraction of correctly selected features (F) with different levels of heritability $\left(h^{2}\right)$ and epistatic effect $(E) . \mathbf{A}, \mathrm{E}=0 ; \mathbf{B}, \mathrm{E}=5 ; \mathbf{C}, \mathrm{E}=10 ; \mathbf{D}, \mathrm{E}=15$. 

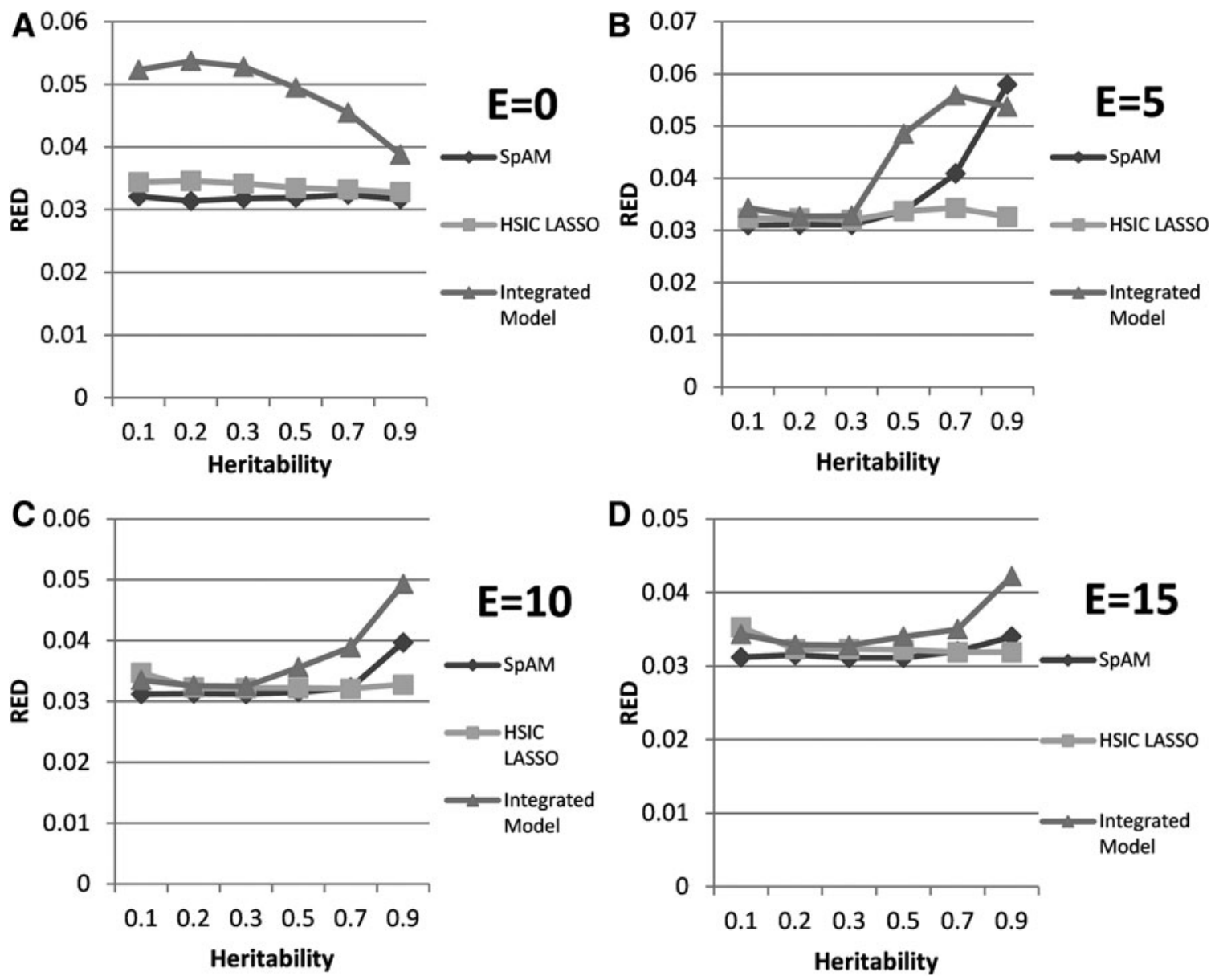

FIG. 3. Comparison of RED with different levels of heritability $\left(h^{2}\right)$ and epistatic effect $(E)$. RED, redundancy rate. $\mathbf{A}, \mathrm{E}=0 ; \mathbf{B}, \mathrm{E}=5 ; \mathbf{C}, \mathrm{E}=10 ; \mathbf{D}, \mathrm{E}=15$.

the redundant markers during estimation of GEBV. Therefore, in terms of RED score, performances of all three methods are excellent. However, the RED score of the proposed model was found to be slightly more than the other two methods (Fig. 3a-d).

\section{DISCUSSION}

Statistical models are extensively used for determination of superior parents for breeding on the basis of their phenotypic performance. With the advent of high-throughput genotyping technology, it becomes possible to estimate GEBV of individuals from the breeding population by combining genotypic and phenotypic information. Statistical models are available for estimation of GEBV for GS. The accuracy of GEBV estimate depends on selection of relevant genetic markers associated with trait of interest. Most of the procedures for identification of these genetic markers are able to capture additive genetic effects; recently, attempts have been made to develop techniques to identify markers that can capture nonadditive genetic effects. It is pertinent to note that the estimation of GEBV of an individual using any of these set of markers, that is, additive or nonadditive may lead to underestimate the genetic potential of an individual. Therefore, it is pertinent to use both categories of markers to estimate GEBV reliably. Considering this, several additive and nonadditive models are evaluated on the basis of their performance for marker identification. These procedures include linear least-squared regression, LASSO, ridge regression, BLUP, mRMR, SpAM, and HSIC LASSO.

In this article, an attempt has been made to integrate selected markers from most efficient additive and nonadditive marker selection procedures available in the literature for GS. From the above results, it can be 
observed that the performance of SpAM, that is, additive effect model, is superior in terms of SE of estimates in the absence of epistatic effect especially at a low heritability level, which is the most practical situation in the real world. It may be noted that in the presence of nonadditive effects, the performance of HSIC LASSO, that is, model for nonadditive effect, is far superior to SpAM due to obvious reasons. In the proposed integrated framework that captures both additive and nonadditive genetic effects, the SE is quite low in comparison with both of these estimates of GEBV as this is derived by optimally combining the estimates from these models. In terms of PA, the integrated framework performs very well in case of dominance of additive effects in the genetic architecture, which is the normally occurring phenomenon in the real world. The proposed integrated model also performs well in terms of fraction of correctly selected features in the presence of moderate and high epistatic effects, which shows that it is robust against nonlinear dominant effects. The performance of all three models in terms of RED score is comparable. This shows that all three models are efficient in selection of independent features, that is, markers. These results are based on empirical comparison through a simulation study, and therefore, there is a need to validate the same on a real data set.

\section{CONCLUSION}

In this article, attempt has been made to identify superior techniques for selection of additive and nonadditive genetic markers among competitive methods. Also, a robust integrated approach is proposed based on selected genetic markers to estimate GEBV, which performs well for both additive and nonadditive genetic architectures. The proposed framework has been developed by optimally combining selected genetic markers obtained from the additive model, that is, SpAM and the nonadditive model, that is, HSIC LASSO, which are found to be better among all other methods considered in this study. The performance of the proposed framework is found to be satisfactory in terms of important evaluation measures, that is, SE, PA, F, and RED in most practical situations of the real world.

\section{ACKNOWLEDGMENTS}

The first author acknowledges the fellowship from ICAR-Indian Agricultural Statistics Research Institute (IASRI) for the PhD program. The facilities provided by ICAR-Indian Agricultural Research Institute and ICAR-IASRI are acknowledged.

\section{AUTHORS' CONTRIBUTIONS}

A.R. conceptualized, guided, and wrote the article. S.G. and D.M. performed the computational and analysis part. All authors read and approved the final article.

\section{AUTHOR DISCLOSURE STATEMENT}

The authors declare that the research was conducted in the absence of any commercial or financial relationships that could be construed as a potential conflict of interest. No competing financial interests exist.

\section{FUNDING INFORMATION}

The authors received no specific funding for this work.

\section{SUPPLEMENTARY MATERIAL}

Supplementary Table S1 


\section{REFERENCES}

Bach, F. 2009. Exploring large feature spaces with hierarchical multiple kernel learning, 105-112. In Advances in Neural Information Processing Systems (NIPS).

Fan, J., Guo, S., and Hao, N. 2012.Variance estimation using refitted cross-validation in ultrahigh dimensional regression. J. R. Stat. Soc. 74, 37-65.

Goeman, J.J. 2010. L1 penalized estimation in the Cox proportional hazards model. Biom. J. 52, 70-84.

Gretton, A., Bousquet, O., Smola, A., et al. 2005. Measuring statistical dependence with Hilbert-Schmidt norms, 63-77. In Jain, S., Simon, H.U., and Tomita, E., eds. Algorithmic Learning Theory (ALT). Springer, Berlin, Heidelberg.

Guha Majumdar, S., Rai, A., and Mishra, D.C. 2019. Identification of genetic markers for increasing agricultural productivity: An empirical study. Indian J. Agr. Sci. 89, 1708-1713.

Henderson, C.R. 1975. Best linear unbiased estimation and prediction under a selection model. Biometrics. 31, $423-447$.

Hoerl, A.E., and Kennard, R.W. 1970a. Ridge regression: Applications to nonorthogonal problems. Technometrics. 12 , 69-82.

Hoerl, A.E., and Kennard, R.W. 1970b. Ridge regression: Biased estimation for nonorthogonal problems. Technometrics. 12, 55-67.

Howard, R., Carriquiry, A.L., and Beavis, W.D. 2014. Parametric and nonparametric statistical methods for genomic selection of traits with additive and epistatic genetic architectures. G3 (Bethesda). 4, 1027-1046.

Kao, C.H., and Zeng, Z.B. 2002. Modeling epistasis of quantitative trait loci using Cockerham's model. Genetics. 160 , $1243-1261$.

Liu, H., Lafferty, J., and Wasserman, L. 2009. Nonparametric regression and classification with joint sparsity constraints, 969-976. In Advances in Neural Information Processing Systems.

Meuwissen, T.H.E., Hayes, B.J., and Goddard, M.E. 2001. Prediction of total genetic value using genome-wide dense marker maps. Genetics. 157, 1819-1829.

Peng, H., Long, F., and Ding, C. 2005. Feature selection based on mutual information: Criteria of max-dependency, max-relevance, and min-redundancy. IEEE Trans. Pattern Anal. Mach. Intell. 27, 1226-1237.

R Core Team. 2017. R: A Language and Environment for Statistical Computing. R Foundation for Statistical Computing, Vienna, Austria. Available at: https://www.R-project.org Last accessed June. 26, 2019.

Raskutti, G., Wainwright, M., and Yu, B. 2012. Minimax-optimal rates for sparse additive models over kernel classes via convex programming. J. Mach. Learn. Res. 13, 389-427.

Ravikumar, P., Lafferty, J., Liu, H., et al. 2009. Sparse additive models. J. R. Stat. Soc. Ser. B (Stat. Methodol.). 71, 1009-1030.

Suzuki, T., and Sugiyama, M. 2013. Fast learning rate of multiple kernel learning: Trade-off between sparsity and smoothness. Ann. Stat. 41, 1381-1405.

Tibshirani, R. 1996. Regression shrinkage and selection via the Lasso. J. R. Stat. Soc. 58, 267-288.

Yamada, M., Jitkrittum, W., Sigal, L., et al. 2014. High-dimensional feature selection by feature-wise Kernelized Lasso. Neural Comput. 26, 185-207.

Yandell, B.S., Mehta, T., Banerjee, S., et al. 2007. R/qtlbim: QTL with Bayesian Interval Mapping in experimental crosses. Bioinformatics. 23, 641-643.

Yandell, B.S., Nengjun, Y., Mehta, T., et al. 2012. qtlbim: QTL Bayesian Interval Mapping. R package version 2.0.5. Available at: http://CRAN.R-project.org/package=qtlbim Last accessed June. 26, 2019.

Zhao, Z., Wang, L., and Li, H. 2010. Efficient spectral feature selection with minimum redundancy, 673-678. AAAI Conference on Artificial Intelligence (AAAI), Atlanta, GA, USA.

Zhao, T., Li, X., Liu, H., et al. 2014. SAM: Sparse Additive Modelling. R package version 1.0.5. Available at: https:// CRAN.R-project.org/package=SAM Last accessed June. 26, 2019.

Address correspondence to: Dr. Anil Rai

Centre for Agricultural Bioinformatics ICAR-Indian Agricultural Statistics Research Institute

Pusa

New Delhi 110012

India

E-mail: anil.rai@icar.gov.in 